\title{
Isothermal Recombinase Polymerase amplification (RPA) of Schistosoma haematobium DNA and oligochromatographic lateral flow detection
}

\author{
A. Rosser ${ }^{1}$, D. Rollinson ${ }^{2}$, M. Forrest ${ }^{3}$ and BL Webster ${ }^{2^{*}}$
}

\begin{abstract}
Background: Accurate diagnosis of urogenital schistosomiasis is vital for surveillance/control programs. Amplification of schistosome DNA in urine by PCR is sensitive and specific but requires infrastructure, financial resources and skilled personnel, often not available in endemic areas. Recombinase Polymerase Amplification (RPA) is an isothermal DNA amplification/detection technology that is simple, rapid, portable and needs few resources.

Findings: Here a Schistosoma haematobium RPA assay was developed and adapted so that DNA amplicons could be detected using oligochromatographic Lateral Flow (LF) strips. The assay successfully amplified S. haematobium DNA at $30-45^{\circ} \mathrm{C}$ in 10 mins and was sensitive to a lower limit of $100 \mathrm{fg}$ of DNA. The assay was also successful with the addition of crude urine, up to $5 \%$ of the total reaction volume. Cross amplification occurred with other schistosome species but not with other common urine microorganisms.

Conclusion: The LF-RPA assay developed here can amplify and detect low levels of S. haematobium DNA. Reactions are rapid, require low temperatures and positive reactions are interpreted using lateral flow strips, reducing the need for infrastructure and resources. This together with an ability to withstand inhibitors within urine makes RPA a promising technology for further development as a molecular diagnostic tool for urogenital schistosomiasis.
\end{abstract}

Keywords: S. haematobium, Diagnostics, Urine, DNA amplification, Isothermal, RPA

\section{Findings}

\section{Introduction}

Schistosoma haematobium is one of three major human schistosome species and causes $>110$ million cases of urogenital schistosomiasis throughout Africa, parts of the Middle East, Madagascar, the Indian Ocean Islands and more recently Corsica. Infection can result in, hematuria, bladder wall pathology, hydronephrosis leading to severe kidney disease [1, 2] and bladder cancer [3]; in addition, an estimated 16 million women suffer from female genital schistosomiasis (FGS), which can cause complications in relation to fertility and HIV transmission [4].

Sensitive and specific diagnostic tests are critical for the development and success of schistosomiasis control and elimination programs. They not only enable the accurate

\footnotetext{
* Correspondence: b.webster@nhm.ac.uk

2Department of Life Sciences, Natural History Museum, London, UK

Full list of author information is available at the end of the article
}

diagnosis and treatment of individual patients but also provide accurate surveillance data necessary to assess the effectiveness of control interventions and to detect resurgence [5]. Moreover, as a control program achieves success, low infection intensity is common increasing the need for improved diagnostic sensitivity and specificity to prevent false negative diagnosis [6-10].

Various modalities for the diagnosis and surveillance of urogenital schistosomiasis are available but none exhibit optimal performance characteristics for sensitive and specific Point-Of-Care (POC) diagnosis [7-12]. Molecular diagnostics that target and amplify schistosome DNA from within urine and stool samples potentially offer high sensitivity and specificity $[7,8,13,14]$. However, these methods are costly, do require significant laboratory infrastructure and technical ability, which can restrict their use in endemic field settings [15].

Recombinase polymerase amplification (RPA) is an isothermal DNA amplification technology offering several 
advantages over other DNA amplification methods especially in terms of its application in more basic laboratory settings in the field. The recombinase polymerase reaction is specific, robust and can take place at an ambient temperature [16, 17]. DNA amplification can be detected by oligochromatographic lateral flow (LF) strips or real time fluorescence, which offers detection flexibility and utility in endemic settings [18]. Here we describe a pilot project investigating the viability of RPA to amplify the $S$. haematobium Dra1 DNA region [19]. The assays specificity and sensitivity were investigated together with its ability to withstand PCR inhibitors found within crude urine.

\section{RPA Assay development}

Suitable (30-35 bp, GC content 30-70 \%) RPA primers were designed (using Primer3 (http://primer3.ut.ee/)), to target $110-120 \mathrm{bp}$ of the $S$. haematobium Dra1 (www.ncbi.nlm.nih.gov/genbank, GenBank: DQ157698.1) DNA region.

Combinations of the different RPA forward and reverse primers were tested for their ability to amplify the Dra1 repeat region using the TwistAmp Basic kit (TwistDx, UK), with slight modifications to the manufacturer's protocol. $1 \mathrm{X}$ rehydration buffer and $\mathrm{dH}_{2} \mathrm{O}$ was added to the dry enzyme pellet and thoroughly mixed. $10 \mu \mathrm{M}$ of each forward and reverse primer was added together with $1 \mathrm{ng}$ of template $S$. haematobium gDNA (Senegal strain from the Schistosomiasis Collection at the Natural History Museum (SCAN) [19]) and the reaction was initiated by addition of $280 \mathrm{mM}$ of magnesium acetate. Reactions were incubated at $37{ }^{\circ} \mathrm{C}$ with constant shaking at $300 \mathrm{rpm}$ in a Minitron shaker (Infors, Switzerland) for $20 \mathrm{~min}$. The reactions were purified with a QIAquick PCR Purification Kit (Qiagen, USA) and then visualised on a $4 \%$ agarose gel. The primer pair, Dra-RPA-F1 + R1 (Table 1), demonstrated the best target amplification (111 bp), producing amplicon bands of increasing size representing multiples of the original target sequence [20] (Fig. 1). The sensitivity of the assay was evaluated with a detection limit of $100 \mathrm{fg}$ of DNA observed (Fig. 1a).

\section{Dra1 LF-RPA assay development}

The Dra1_RPA_F1 + R1 primers were adapted for the LFRPA assay by labelling of the reverse primer with a $5^{\prime}$ biotin residue and an internal RPA LF probe (Dra1 LF probe) was designed according to the TwistDX guidelines (Table 1). $5 \mu \mathrm{M}$ of each LF primer (Dra1-LF-F1 + R1), $5 \mu \mathrm{M}$ of the Dra1 LF probe $(10 \mu \mathrm{M}), 0.25 \mathrm{M}$ betaine (Sigma Aldrich, UK) was mixed with $1 \times$ rehydration buffer, $\mathrm{dH} 2 \mathrm{O}$ and was added to the reaction pellet of the TwistAmp nfo kit (TwistDX, UK). $1 \mathrm{ng}$ of the template S. haematobium DNA was added and the reaction was initiated by addition of $280 \mathrm{mM}$ of magnesium acetate. Reactions were incubated at $37{ }^{\circ} \mathrm{C}$ in a Minitron shaker at $300 \mathrm{rpm}$ for $20 \mathrm{~min}$. After incubation, $1 \mu \mathrm{l}$ of the amplification was diluted in the running buffer (Milenia Biotec, Germany) and $10 \mu \mathrm{l}$ placed on the sample pad of a Genline Hybridetect -1 LF strip (Milenia Biotec, Germany). The strips were placed vertically into separate tubes containing $100 \mu \mathrm{l}$ of running buffer and incubated at room temperature with the final result read at $5 \mathrm{~min}$.

Detection of the Dra1 LF-RPA amplicons was observed as a positive test line on the LF strips (Fig. 1b). Faint test bands in the negative controls were sometimes observed if the LF strips were left for too long before being read, creating false positives. Increasing the amplicon dilution in the running buffer to a ratio of 1 in 100 from the recommended 1 in 50 abrogated this problem. The sensitivity of Dra1 LF-RPA assay was evaluated with a limit of detection observed at $100 \mathrm{fg}$ of DNA (Fig. 1b). The effect of varying reaction temperatures and times were also assessed. The assay was found to operate over a temperature range of $30-45{ }^{\circ} \mathrm{C}$, with reduced amplification at lower temperatures and no amplification at $50{ }^{\circ} \mathrm{C}$ (Fig. 2a). Amplification was detectable after as little as $5 \mathrm{~min}$ of incubation and optimal amplification was reached at $10 \mathrm{~min}$ (Fig. 2b).

\section{Effect of crude urine}

Increasing volumes $(0.31 \mu \mathrm{l}, 0.63 \mu \mathrm{l}, 1.25 \mu \mathrm{l}$ and $2.50 \mu \mathrm{l})$ (Table 2) of donor urine were spiked with $100 \mathrm{fg}$ and also $10 \mathrm{pg}$ of $S$. haematobium gDNA before being added to the Dra1 LF-RPA reactions. Each reaction volume was adjusted

Table 1 Primers / probe sequence design for the Dra1 RPA and LF assays

\begin{tabular}{lll}
\hline Assay & Name & Sequence $\left(5^{\prime}{ }^{\prime}{ }^{\prime}{ }^{\prime}\right)$ \\
\hline RPA & Dra1-F1 & ATCTCACCTATCAGACGAAACAAAGAAAAT \\
& Dra1-R1 & AATATGAAACAATITCACAACGATACGAC \\
LF-RPA & Dra1-F1-LF & ATCTCACCTATCAGACGAAACAAAGAAAAT \\
& Dra1-R1-LF & (Z)AATATGAAACAATTTCACAACGATACGAC \\
& Dra1 LF probe & (Y)AATTGTTGGTGAAGTGCCTGTTCGCAAT(H)TCTCCGGAATGGTTG(3)
\end{tabular}

Features: $\mathbf{Z}=$ Biotin label; $\mathbf{Y}=6$-carboxyfluorescein (FAM) label; $\mathbf{H}=$ abasic tetrahydrofuran (THF) residue; $\mathbf{3}=\mathrm{C} 3$ spacer. All primers were designed according to the instructions from TwistDx (http://www.twistdx.co.uk). The Dra1 LF probe was designed with a recommended length of 46-52 bp with at least 30 bp placed 5' to the abasic tetrahydrofuran (THF) residue (H). The RPA and LF RPA primers are the same but with a $5^{\prime}$ modification on the reverse primer (Dra1-R1-LF) for the LF RPA assay 

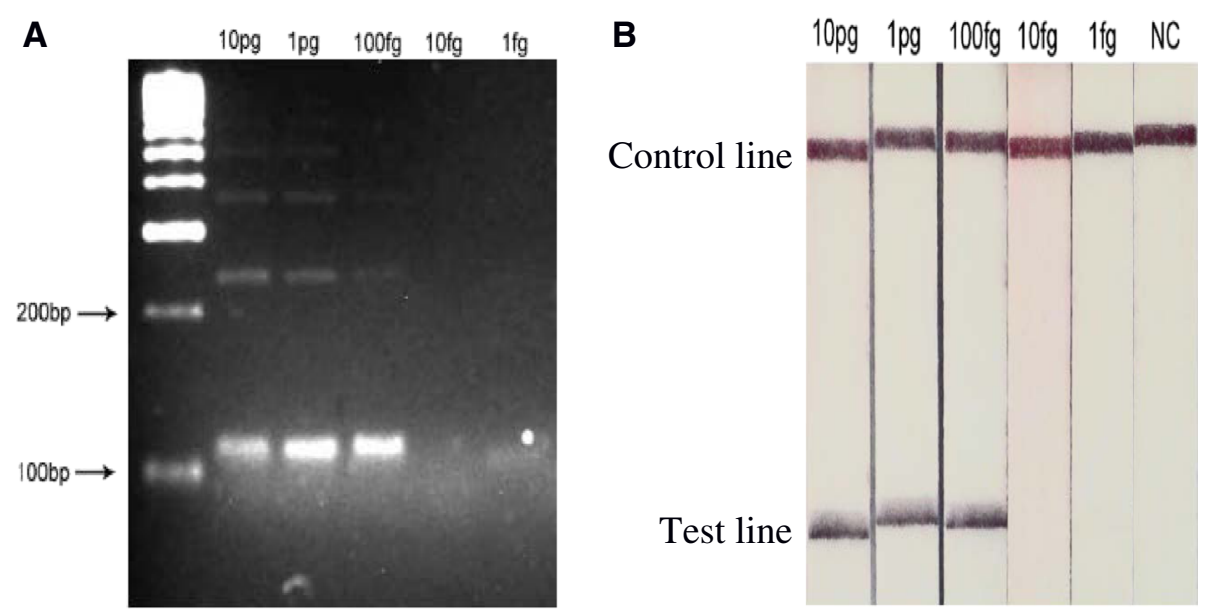

Fig. 1 a Agarose gel image of the Dra1 RPA amplicons. Low limit of detection can be seen at $100 \mathrm{fg}$ of $\mathrm{S}$. haematobium gDNA. $\mathbf{b}$ LF strips showing the detection of the Dra LF-RPA amplicons. Lower limit of detection can be seen at $100 \mathrm{fg}$ of S. haematobium gDNA. NC=Negative control. The LF-RPA oligonucleotides consisted of a forward primer, a specialised 6-FAM labelled oligonucleotide probe and a reverse biotin labelled primer. Upon successful binding to the complementary gDNA target, amplification ensues resulting in the formation of a double-labelled amplicon. When run on an oligochromatographic LF strip, the amplicon binds to anti-FAM antibodies and antibody labelled gold colloid nanoparticles in the running buffer bind to the biotin antigen resulting in a semi quantitative colour change. The LF strip also has a control line to test for reaction failure

to a final volume of $25 \mu \mathrm{l}$ with $\mathrm{H}_{2} \mathrm{O}$ so that the percentage of crude urine within the reactions ranged from 1 to $10 \%$. With $100 \mathrm{fg}$ of S. haematobium gDNA the addition of $1.25 \%$ urine had no impact upon amplification efficacy. A marked reduction in amplification efficacy was qualitatively noted at $2.5 \%$ and $5 \%$ of total reaction volume and no amplification was visible at $10 \%$. In contrast gDNA levels of $10 \mathrm{pg}$ increased the assays efficacy with significant amplification being detected even at $10 \%$ of total reaction volume of urine.

\section{Specificity}

Cross amplification was observed with 1 pg of S. curassoni and $S$. bovis DNA and at a higher limit of $100 \mathrm{pg}$ for $S$. mansoni (samples supplied by SCAN [19]). No cross amplification was seen with Escherichia coli, Klebsiella pneumoniae, Proteus mirabilis (cultures supplied by the Microbiology Department, University Hospitals of Leicester) and human gDNA (www.promega.com).

\section{Discussion}

The detection of species specific gDNA from urine is the sensitive and specific current marker of true $S$. haematobium infection [21]. RPA is an alternative gDNA based diagnostic tool that overcomes several of the obstacles encountered by traditional gDNA based diagnostics [16-18], particularly suiting it to Point-Of-Care (POC) use [22]. All reagents are readily available lyophilized with the main RPA reagents provided in a single dried pellet, which simplifies assay preparation and allows easy transportation and long-term storage at room temperature. DNA amplification is quick, requiring low ambient temperatures and
A

Control line

Test line

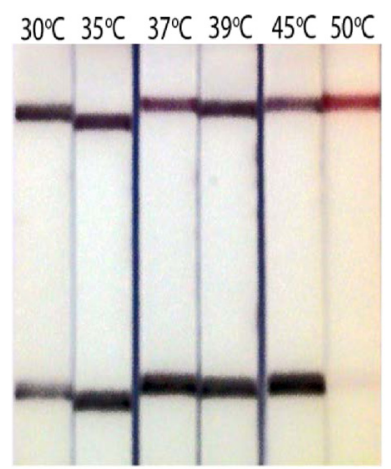

B

Control line

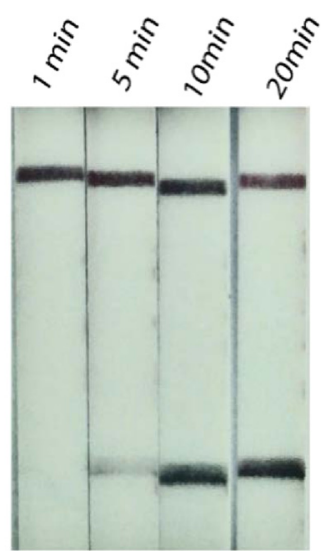

Fig. 2 LF strips showing Dra1 LF-RPA amplicon detection at different reaction temperatures (a) and times (b) 
Table 2 Results showing the impact of crude urine on the efficacy of the Dra1 RPA-LF assay and subsequent LF detection. All reactions were performed at $37^{\circ} \mathrm{C}$ for 20 mins

\begin{tabular}{|c|c|c|c|}
\hline \multicolumn{2}{|c|}{ Measure of crude urine in each Dra1 RPA-LF reaction } & \multirow{2}{*}{\multicolumn{2}{|c|}{$\begin{array}{l}\text { Dra1 RPA-LF efficacy } \\
\text { Amount of S. haematobium gDNA }\end{array}$}} \\
\hline \multirow[t]{2}{*}{ Volume of crude urine $(\mu \mathrm{l})$} & \multirow[t]{2}{*}{$\%$ of crude urine } & & \\
\hline & & $10 \mathrm{pg}$ & $100 \mathrm{fg}$ \\
\hline 0.31 & $1.25 \%$ & +++ & +++ \\
\hline 0.63 & $2.5 \%$ & +++ & ++ \\
\hline 1.25 & $5 \%$ & +++ & + \\
\hline 2.50 & $10 \%$ & +++ & - \\
\hline
\end{tabular}

The number of + refers to the qualitative signal on the LF test line. $+++=$ very strong and - = no trace

this together with the LF detection system is very simple and portable to use, with results easily interpreted by personnel with little training [16-18].

Here, RPA was successfully developed to amplify the Dra1 DNA region of $S$. haematobium and amplification success was easily detected by a semi quantitative colourimetric change on LF strips. Amplification was detectable after only $5 \mathrm{~min}$ of incubation and at $30^{\circ} \mathrm{C}$, thus incubation using human body heat, ambient environmental temperatures in endemic areas or battery powered devices would suffice, removing the need for external power supplies and infrastructure [22].

The current LF-RPA sensitivity was lower than existing Dra1 amplification assays [21]. However, theoretically RPA can detect single template DNA copies thus there is scope for further LF-RPA optimisation and development to increase the sensitivity of the assay to achieve that achieved with RT-PCR and LAMP [21]. RPA offers degrees of tolerance to inhibitors, such as those found in urine [17] suggesting direct testing of untreated urine from infected people will be feasible. The RPA's reaction mechanisms and chemistry means that total purification of total DNA from samples is not required [17], reducing the need for expensive, technical and time-consuming sample DNA preparation / purification, enhancing its feasibility in a POC situation [23]. The current cost per test is high at approximately $\$ 5$ (reaction + detection) however, RPA is a newly developed technique and prices are likely to decrease in the future while availability and throughput will increase. For example centrifugal microfluidic cartridges that can split a single reaction to enable testing of eight individual samples have been developed [22] reducing the cost to $\$ 0.6$.

The Dra1 LF-RPA specificity to schistosome DNA was high with no cross-reaction with Enterobacteria or human gDNA. As found previously with RT-PCR [24], RPA Dra1 cross-amplification with S. mansoni was 1000 fold less sensitive than for $S$. haematobium but as predicted crossamplification was successful with $S$. curassoni and S. bovis, both members of the $S$. haematobium group, known to possess the Dra1 repeat sequence [25]. Thus there is potential to utilise this assay and / or develop alternative species-specific assays for the identification of other $S$. haematobium-group species infections in livestock and humans.

In conclusion, the Dra1 LF-RPA assay developed here provides a robust $S$. haematobium DNA amplification system offering advantages over currently available DNA diagnostic systems; requiring minimal infra structure, equipment, technical support, sample preparation and is tolerant to inhibitors in crude urine. With further development this assay has potential for POC diagnosis of urogenital schistosomiasis in endemic field settings.

\section{Abbreviations}

RPA: Recombinase polymerase amplification; LF: Lateral flow; gDNA: Genomic deoxyribonucleic acid; POC: Point-of-care; PCR: Polymerase chain reaction; RT-PCR: Real time PCR.

\section{Competing interests}

The authors declare that they have no competing interests.

\section{Authors' contributions}

BW conceived the study. AR helped develop the project and carried out the practical / laboratory work as part of his masters research project. BW, AR and DR wrote the manuscript. DR and BW collected the original reference samples made available by SCAN. MF advised us on various aspects with regard to the feasibility of the study and touble-shooting needed during the development of the assay. All authors read and approved the final version of the manuscript.

\section{Acknowledgements}

This study was financially supported by the London School of Hygiene and Tropical Medicine masters project bench fees and the NHM Life Sciences Departmental Investment Fund. This publication and BW were supported by the University of Georgia Research Foundation, which is funded by the Bill and Melinda Gates Foundation for the SCORE project (http://score.uga.edu), (Prime award number 50816, sub-award numbers: RR374-053/4785426). The authors would also like to thank Dr Aidan Emery and Dr Fiona Allen for helpful discussions and advice.

\section{Author details}

${ }^{1}$ Faculty of Infectious and Tropical Diseases, London School of Hygiene and Tropical Medicine, London, UK. ²Department of Life Sciences, Natural History Museum, London, UK. ${ }^{3}$ TwistDX Ltd, Cambridge, UK.

Received: 17 August 2015 Accepted: 25 August 2015

Published online: 04 September 2015

\section{References}

1. van der Werf MJ, de Vlas SJ, Brooker S, Looman CW, Nagelkerke NJ, Habbema JD, et al. Quantification of clinical morbidity associated with schistosome infection in sub-Saharan Africa. Acta trop. 2003;86(2):125-39. 
2. Rollinson D. A wake up call for urinary schistosomiasis: reconciling research effort and public health importance. Parasitol. 2009;136:1593-610.

3. Shiff C, Veltri R, Naples J, Quartey J, Otchere J, Anyan W, et al. Ultrasound verification of bladder damage is associated with known biomarkers of bladder cancer in adults chronically infected with Schistosoma haematobium in Ghana. Trans Roy Soc Trop Med Hyg. 2006;100(9):847-54.

4. Kjetland EF, Norseth HM, Taylor M, Lillebø K, Kleppa E, Holmen SD, et al. Classification of the lesions observed in female genital schistosomiasis. Int J Gyn Obs. 2014;127:227-8.

5. Bergquist $\mathrm{R}$, Johansen MV, Utzinger J. Diagnostic dilemmas in helminthology: what tools to use and when? Trends Parasitol. 2009;25:151-6.

6. Rollinson D, Knopp S, Levitz S, Stothard JR, Tchuem Tchuente LA, Garba A, et al. Time to set the agenda for schistosomiasis elimination. Acta Trop. 2013;128:423-40.

7. Cavalcanti MG, Silva LF, Peralta RH, Barreto MG, Peralta JM. Schistosomiasis in areas of low endemicity: a new era in diagnosis. T Parasitol. 2013;29:75-82.

8. Stothard JR, Stanton MC, Bustinduy AL, Sousa-Figueiredo JC, Van Dam GJ, Betson M, et al. Diagnostics for schistosomiasis in Africa and Arabia: a review of present options in control and future needs for elimination. Parasitol. 2014;141:1947-61.

9. King $\mathrm{CH}$, Bertsch D. Meta-analysis of urine heme dipstick diagnosis of Schistosoma haematobium infection, including low-prevalence and previously-treated populations. PLoS Neg Trop Dis. 2013;7:e2431.

10. McCarthy JS, Lustigman S, Yang GJ, Barakat RM, Garcia HH, Sripa B, et al. A research agenda for helminth diseases of humans: diagnostics for control and elimination programmes. PLoS Neg Trop Dis. 2012;6:e1601.

11. Stothard JR, Sousa-Figueiredo JC, Standley C, Van Dam GJ, Knopp S, Utzinger J, et al. An evaluation of urine-CCA strip test and fingerprick blood SEA-ELISA for detection of urinary schistosomiasis in schoolchildren in Zanzibar. Acta Trop. 2009;111:64-70.

12. Nausch N, Dawson EM, Midzi N, Mduluza T, Mutapi F, Doenhoff MJ. Field evaluation of a new antibody-based diagnostic for Schistosoma haematobium and S. mansoni at the point-of-care in northeast Zimbabwe. BMC Infect Dis. 2014;14:165.

13. Obeng BB, Aryeetey YA, de Dood CJ, Amoah AS, Larbi IA, Deelder AM, et al. Application of a circulating-cathodic-antigen (CCA) strip test and real-time $P C R$, in comparison with microscopy, for the detection of Schistosoma haematobium in urine samples from Ghana. Ann Trop Med Parasitol. 2008;102:625-33.

14. Vinkeles Melchers NV, van Dam GJ, Shaproski D, Kahama Al, Brienen EA, Vennervald BJ, et al. Diagnostic performance of Schistosoma real-time PCR in urine samples from Kenyan children infected with Schistosoma haematobium: day-to-day variation and follow-up after praziquantel treatment. PLoS Neg Trop Dis. 2014;8:e2807.

15. Pai NP, Vadnais C, Denkinger C, Engel N, Pai M. Point-of-care testing for infectious diseases: diversity, complexity, and barriers in low- and middle-income countries. PLoS Medicine. 2012;9:e1001306.

16. Ahmed A, van der Linden $H$, Hartskeerl RA. Development of a recombinase polymerase amplification assay for the detection of pathogenic Leptospira. Int J En Res Pub Health. 2014;11:4953-64.

17. Krolov K, Frolova J, Tudoran O, Suhorutsenko J, Lehto T, et al. Sensitive and rapid detection of Chlamydia trachomatis by recombinase polymerase amplification directly from urine samples. J Mol Diag. 2014;16:127-35

18. Kersting S, Rausch V, Bier FF, von Nickisch-Rosenegk M. Rapid detection of Plasmodium falciparum with isothermal recombinase polymerase amplification and lateral flow analysis. Mal J. 2014;13(1):99.

19. Emery AM, Allan FE, Rabone ME, Rollinson D. Schistosomiasis collection at NHM (SCAN). Para Vect. 2012;5:185

20. Amarir F, Sebti F, Abbasi I, Sadak A, Fellah H, Nhammi H, et al. Schistosoma haematobium detection in snails by Dral PCR and Sh110/Sm-SI PCR: further evidence of the interruption of schistosomiasis transmission in Morocco. Para Vect. 2014;7:288

21. Lodh N, Naples JM, Bosompem KM, Quartey J, Shiff CJ. Detection of parasite-specific DNA in urine sediment obtained by filtration differentiates between single and mixed infections of Schistosoma mansoni and S. haematobium from endemic areas in Ghana. PLoS One. 2014;9:e91144.

22. Escadafal C, Faye O, Sall AA, Faye O, Weidmann M, Strohmeier O, et al. Rapid molecular assays for the detection of yellow fever virus in low-resource settings. PLoS Neg Trop Dis. 2014;8:e2730.
23. Wichmann D, Panning M, Quack T, Kramme S, Burchard GD, Grevelding C, et al. Diagnosing schistosomiasis by detection of cell-free parasite DNA in human plasma. PLoS Neg Trop Dis. 2009;3:e422.

24. Cnops L, Soentjens P, Clerinx J, Van Esbroeck M. A Schistosoma haematobium-specific real-time PCR for diagnosis of urogenital schistosomiasis in serum samples of international travelers and migrants. PLoS Neg Trop Dis. 2013;7:e2413.

25. Akinwale OP, Hock TT, Chia-Kwung F, Zheng Q, Haimo S, Ezeh C, et al. Differentiating Schistosoma haematobium from Schistosoma magrebowie and other closely related schistosomes by polymerase chain reaction amplification of a species specific mitochondrial gene. Trop Parasitol. 2014:4:38-42.

\section{Submit your next manuscript to BioMed Central and take full advantage of:}

- Convenient online submission

- Thorough peer review

- No space constraints or color figure charges

- Immediate publication on acceptance

- Inclusion in PubMed, CAS, Scopus and Google Scholar

- Research which is freely available for redistribution 